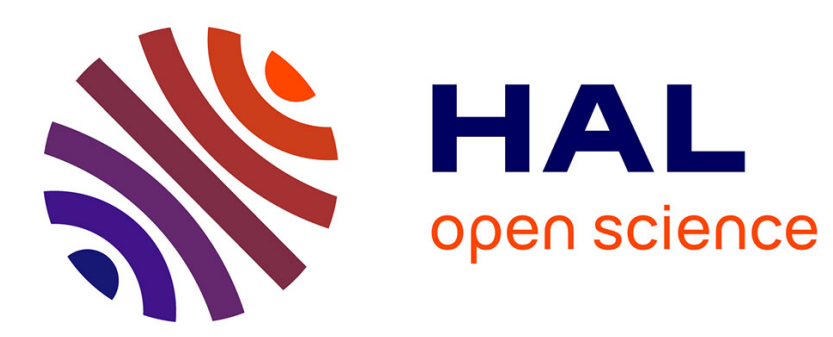

\title{
Benchmarking the $(1+1)-E S$ with One-Fifth Success rule on the BBOB-2009 Noisy Testbed
}

\author{
Anne Auger
}

\section{To cite this version:}

Anne Auger. Benchmarking the (1+1)-ES with One-Fifth Success rule on the BBOB-2009 Noisy Testbed. ACM-GECCO Genetic and Evolutionary Computation Conference, Jul 2009, Montreal, Canada. inria-00430516

\section{HAL Id: inria-00430516 https://hal.inria.fr/inria-00430516}

Submitted on 8 Nov 2009

HAL is a multi-disciplinary open access archive for the deposit and dissemination of scientific research documents, whether they are published or not. The documents may come from teaching and research institutions in France or abroad, or from public or private research centers.
L'archive ouverte pluridisciplinaire HAL, est destinée au dépôt et à la diffusion de documents scientifiques de niveau recherche, publiés ou non, émanant des établissements d'enseignement et de recherche français ou étrangers, des laboratoires publics ou privés. 


\title{
Benchmarking the (1+1)-ES with One-Fifth Success rule on the BBOB-2009 Noisy Testbed
}

\author{
Anne Auger \\ TAO Team, INRIA Saclay lle-de-France \\ LRI, Bat 490 Univ. Paris-Sud \\ 91405 Orsay Cedex France \\ Anne.Auger@inria.fr
}

\begin{abstract}
The (1+1)-ES with one-fifth success rule is one of the first and simplest stochastic algorithm proposed for optimization on a continuous search space in a black-box scenario. In this paper, we benchmark an independent-restart (1+1)-ES with one-fifth success rule on the BBOB-2009 noisy testbed. The maximum number of function evaluations used equals $10^{6}$ times the dimension of the search space. The algorithm could only solve 3 functions with moderate noise in 5-D and 2 functions in 20-D.
\end{abstract}

\section{Categories and Subject Descriptors}

G.1.6 [Numerical Analysis]: Optimization-global optimization, unconstrained optimization; F.2.1 [Analysis of Algorithms and Problem Complexity]: Numerical Algorithms and Problems

\section{General Terms}

Algorithms

\section{Keywords}

Benchmarking, Black-box optimization, Evolutionary computation

\section{INTRODUCTION}

The (1+1)-ES with one-fifth success rule is one of the earliest and simplest adaptive stochastic search algorithm $[8$, $7,3]$. This paper complements [1] where an independentrestart implementation of the (1+1)-ES with one-fifth success rule is benchmarked on the BBOB-2009 noise-free testbed. Indeed, we test exactly the same algorithm, using the same settings on the BBOB-2009 noisy testbed. For the description of the algorithm and the settings we refer to [1].

Permission to make digital or hard copies of all or part of this work for personal or classroom use is granted without fee provided that copies are not made or distributed for profit or commercial advantage and that copies bear this notice and the full citation on the first page. To copy otherwise, to republish, to post on servers or to redistribute to lists, requires prior specific permission and/or a fee.

GECCO'09, July 8-12, 2009, Montréal Québec, Canada.

Copyright 2009 ACM 978-1-60558-505-5/09/07 ...\$5.00.

\section{RESULTS AND DISCUSSION}

Results from experiments according to [5] on the benchmark functions given in $[4,6]$ are presented in Figures 1 and 2 and in Tables 1 and 2.

We observe that globally the algorithm performs poorly. In 5-D, only $f_{101}, f_{102}, f_{103}$ are solved and in 20-D only $f_{101}$ and $f_{102}$ are solved. The functions solved belong to the class of functions with moderate noise.

\section{Acknowledgments}

The author would like to acknowledge Nikolaus Hansen, Steffen Finck and Raymond Ros for their great and hard work on the BBOB project.

\section{REFERENCES}

[1] A. Auger. Benchmarking the (1+1) Evolution Strategy with One-Fifth Success Rule on the BBOB-2009 Function Testbed. In Workshop Proceedings of the Genetic and Evolutionary Computation Conference (GECCO 2009). ACM Press, 2009.

[2] A. Auger and N. Hansen. Benchmarking the (1+1)-CMA-ES on the BBOB-2009 Function Testbed. In Workshop Proceedings of the Genetic and Evolutionary Computation Conference (GECCO 2009). ACM Press, 2009.

[3] L. Devroye. The compund random search. In International Symposium on Systems Engineering and Analysis, pages 195-110. Purdue University, 1972.

[4] S. Finck, N. Hansen, R. Ros, and A. Auger. Real-parameter black-box optimization benchmarking 2009: Presentation of the noisy functions. Technical Report 2009/21, Research Center PPE, 2009.

[5] N. Hansen, A. Auger, S. Finck, and R. Ros. Real-parameter black-box optimization benchmarking 2009: Experimental setup. Technical Report RR-6828, INRIA, 2009.

[6] N. Hansen, S. Finck, R. Ros, and A. Auger. Real-parameter black-box optimization benchmarking 2009: Noisy functions definitions. Technical Report RR-6869, INRIA, 2009.

[7] I. Rechenberg. Evolutionsstrategie. Friedrich Frommann Verlag (Günther Holzboog KG), Stuttgart, 1973.

[8] M. Schumer and K. Steiglitz. Adaptive step size random search. Automatic Control, IEEE Transactions on, 13:270-276, 1968. 

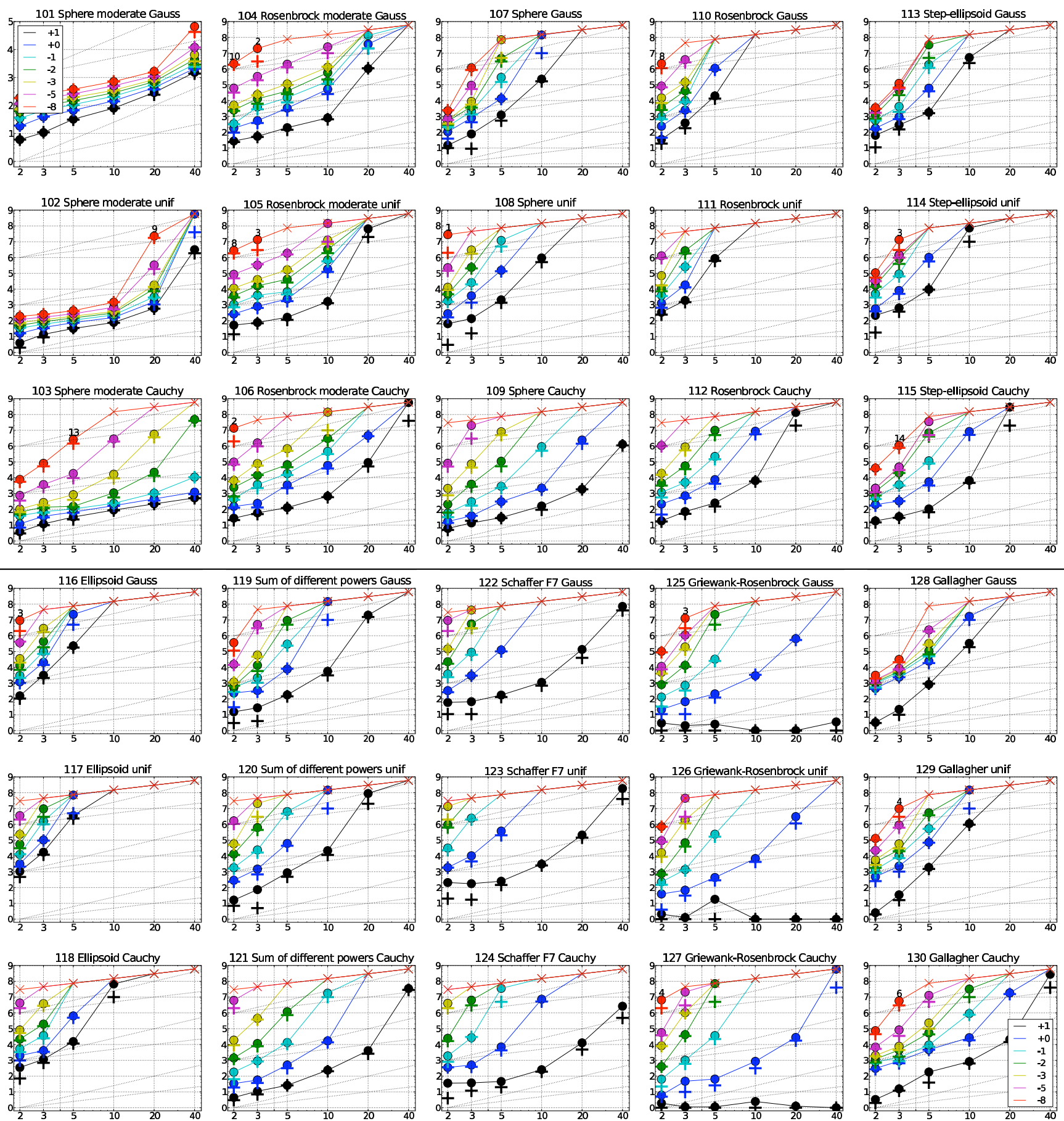

Figure 1: Expected Running Time (ERT, $\bullet$ ) to reach $f_{\mathrm{opt}}+\Delta f$ and median number of function evaluations of successful trials $(+)$, shown for $\Delta f=10,1,10^{-1}, 10^{-2}, 10^{-3}, 10^{-5}, 10^{-8}$ (the exponent is given in the legend of $f_{101}$ and $\left.f_{130}\right)$ versus dimension in $\log -\log$ presentation. The $\operatorname{ERT}(\Delta f)$ equals to $\# \mathrm{FEs}(\Delta f)$ divided by the number of successful trials, where a trial is successful if $f_{\mathrm{opt}}+\Delta f$ was surpassed during the trial. The \# FEs $(\Delta f)$ are the total number of function evaluations while $f_{\mathrm{opt}}+\Delta f$ was not surpassed during the trial from all respective trials (successful and unsuccessful), and $f_{\mathrm{opt}}$ denotes the optimal function value. Crosses $(\times)$ indicate the total number of function evaluations \#FEs $(-\infty)$. Numbers above ERT-symbols indicate the number of successful trials. Annotated numbers on the ordinate are decimal logarithms. Additional grid lines show linear and quadratic scaling. 


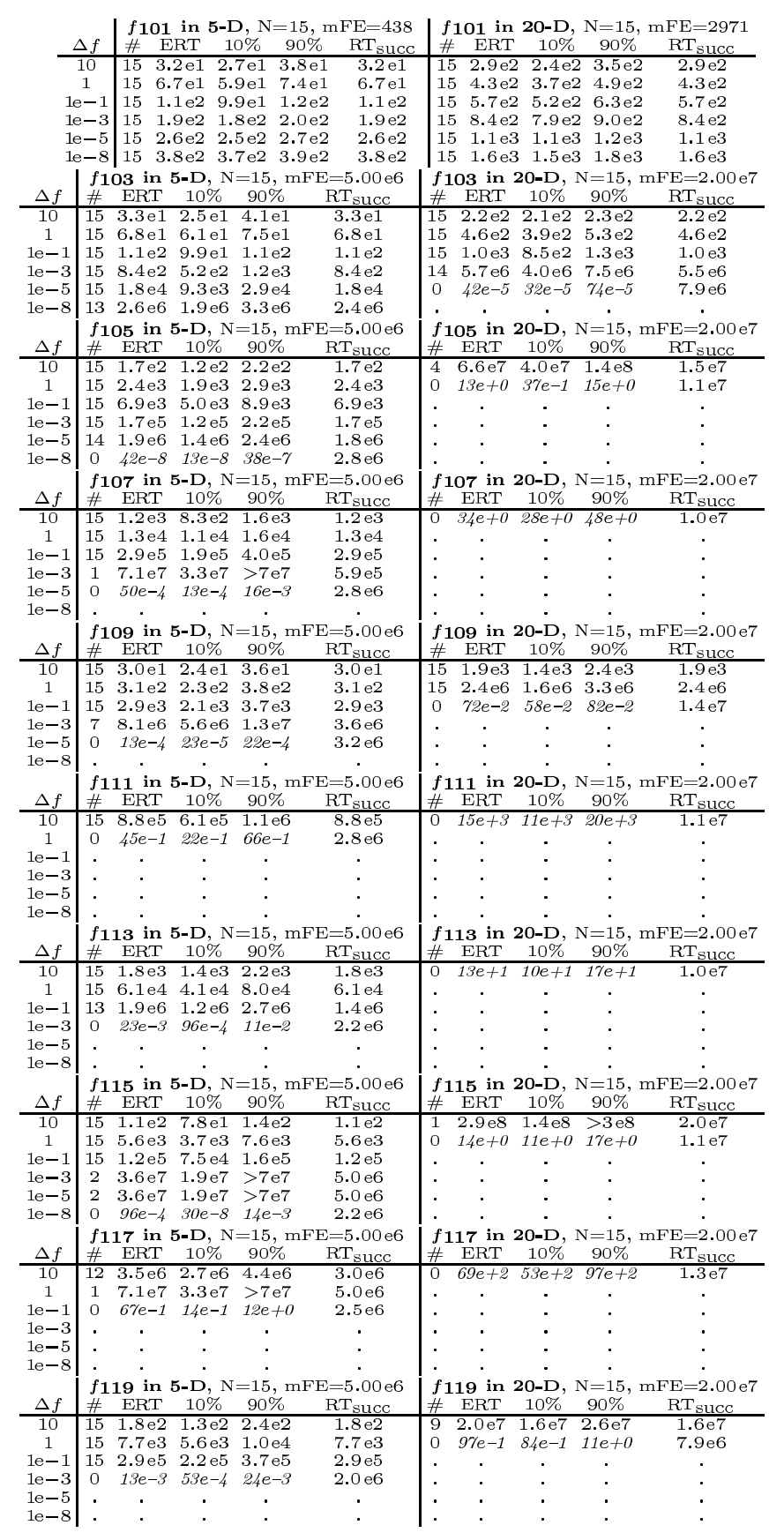

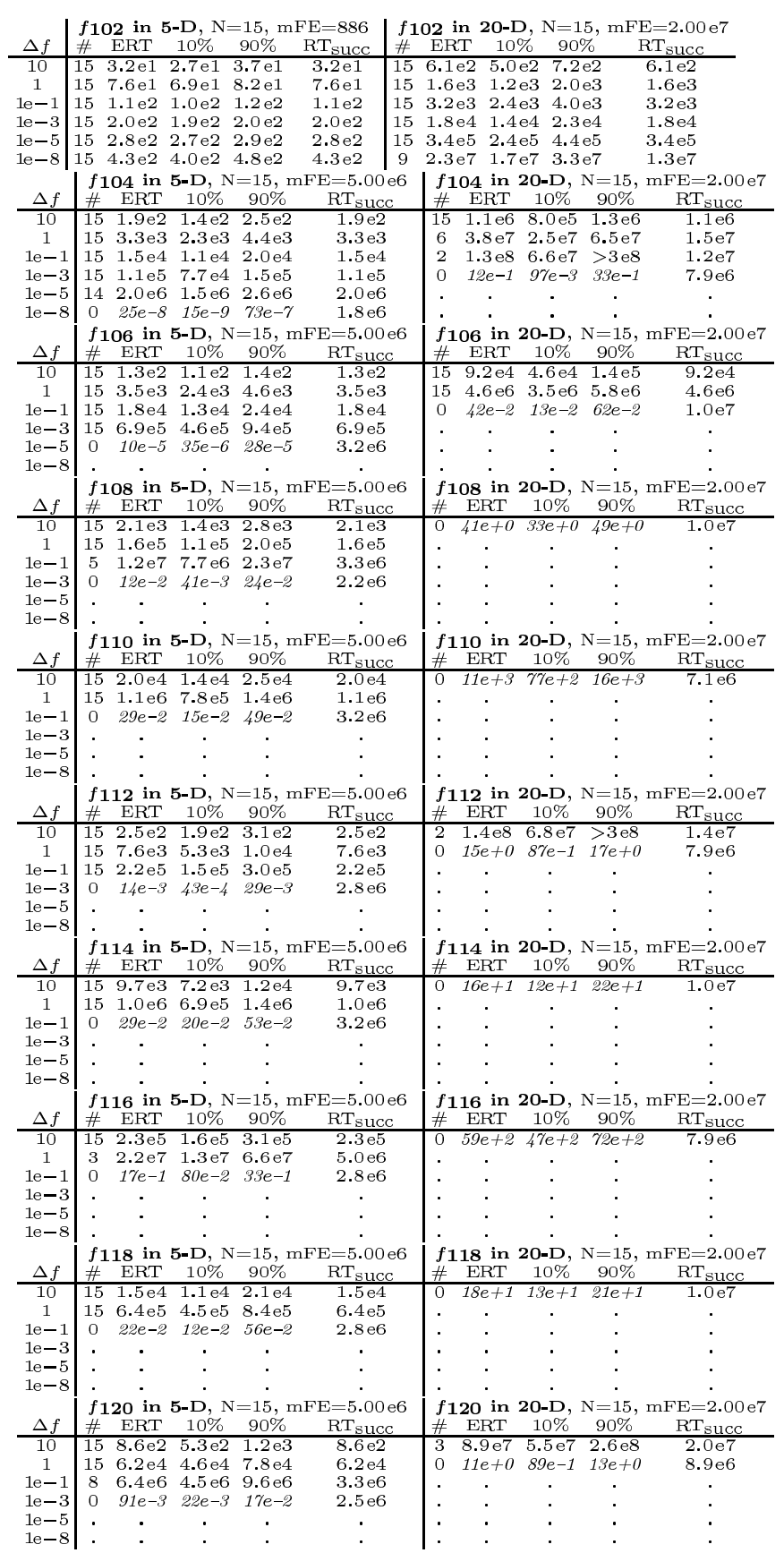

Table 1: Shown are, for functions $f_{101}-f_{120}$ and for a given target difference to the optimal function value $\Delta f$ : the number of successful trials (\#); the expected running time to surpass $f_{\mathrm{opt}}+\Delta f$ (ERT, see Figure 1); the $10 \%$-tile and $90 \%$-tile of the bootstrap distribution of ERT; the average number of function evaluations in successful trials or, if none was successful, as last entry the median number of function evaluations to reach the best function value $\left(\mathrm{RT}_{\text {succ }}\right)$. If $f_{\mathrm{opt}}+\Delta f$ was never reached, figures in italics denote the best achieved $\Delta f$-value of the median trial and the $10 \%$ and $90 \%$-tile trial. Furthermore, $\mathbf{N}$ denotes the number of trials, and $\mathrm{mFE}$ denotes the maximum of number of function evaluations executed in one trial. See Figure 1 for the names of functions. 

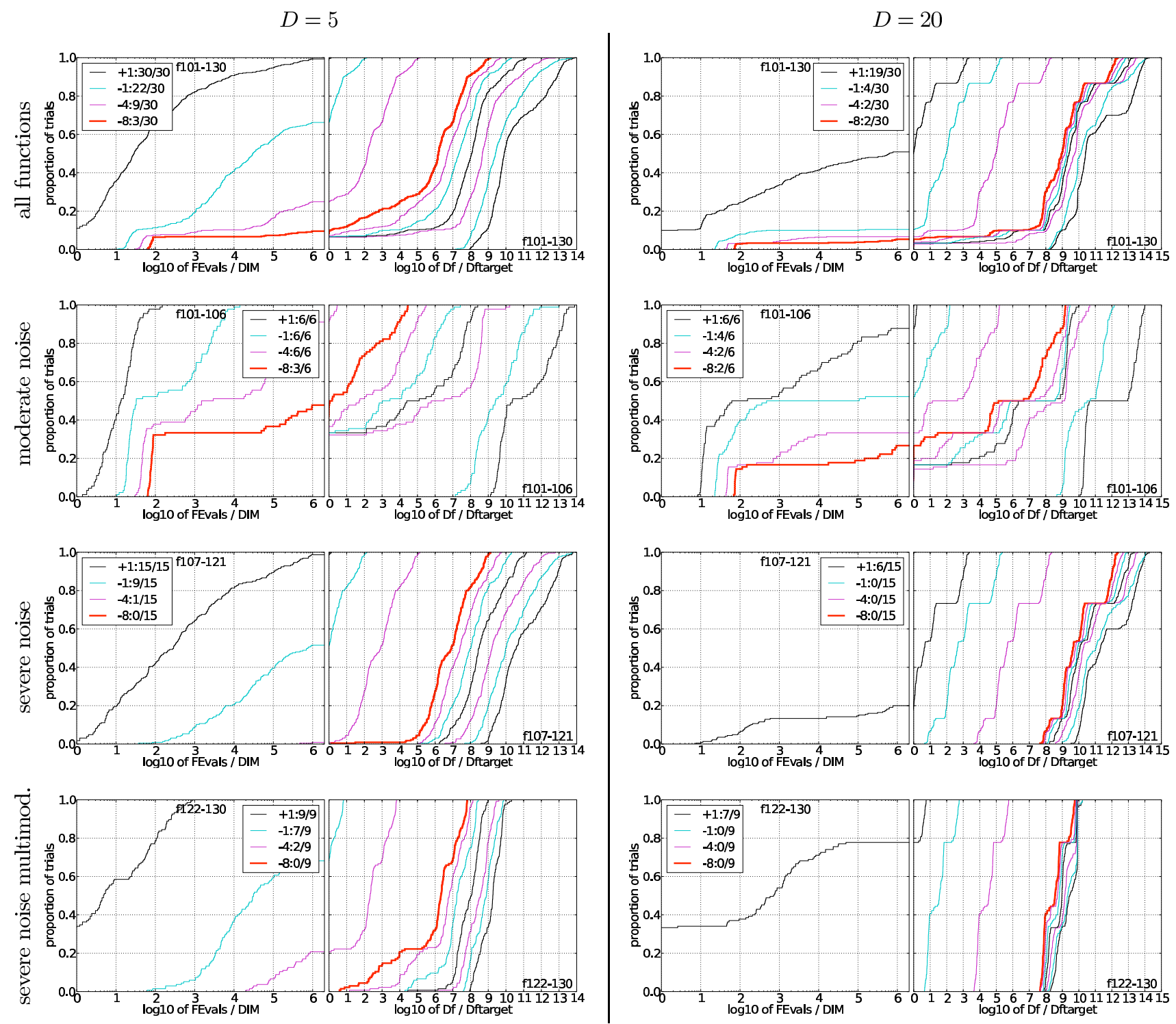

Figure 2: Empirical cumulative distribution functions (ECDFs), plotting the fraction of trials versus running time (left subplots) or versus $\Delta f$ (right subplots). The thick red line represents the best achieved results. Left subplots: ECDF of the running time (number of function evaluations), divided by search space dimension $D$, to fall below $f_{\mathrm{opt}}+\Delta f$ with $\Delta f=10^{k}$, where $k$ is the first value in the legend. Right subplots: ECDF of the best achieved $\Delta f$ divided by $10^{k}$ (upper left lines in continuation of the left subplot), and best achieved $\Delta f$ divided by $10^{-8}$ for running times of $D, 10 D, 100 \mathrm{D} \ldots$ function evaluations (from right to left cycling blackcyan-magenta). Top row: all results from all functions; second row: moderate noise functions; third row: severe noise functions; fourth row: severe noise and highly-multimodal functions. The legends indicate the number of functions that were solved in at least one trial. FEvals denotes number of function evaluations, $D$ and DIM denote search space dimension, and $\Delta f$ and Df denote the difference to the optimal function value. 

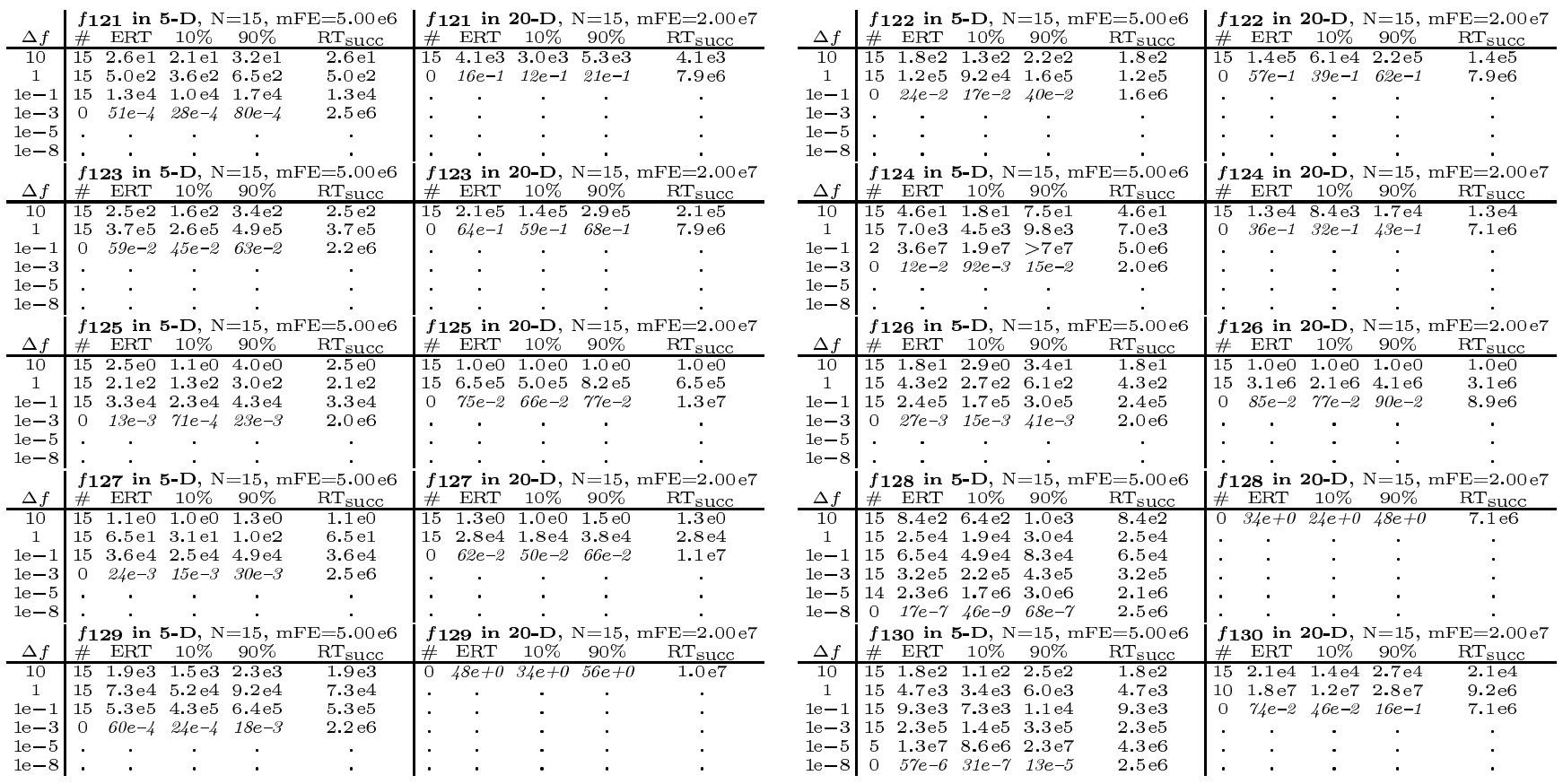

Table 2: Shown are, for functions $f_{121}-f_{130}$ and for a given target difference to the optimal function value $\Delta f$ : the number of successful trials (\#); the expected running time to surpass $f_{\mathrm{opt}}+\Delta f$ (ERT, see Figure 1); the $10 \%$-tile and $90 \%$-tile of the bootstrap distribution of ERT; the average number of function evaluations in successful trials or, if none was successful, as last entry the median number of function evaluations to reach the best function value $\left(\mathrm{RT}_{\text {succ }}\right)$. If $f_{\mathrm{opt}}+\Delta f$ was never reached, figures in italics denote the best achieved $\Delta f$-value of the median trial and the $10 \%$ and $90 \%$-tile trial. Furthermore, $\mathbf{N}$ denotes the number of trials, and $\mathrm{mFE}$ denotes the maximum of number of function evaluations executed in one trial. See Figure 1 for the names of functions. 